Indian J Anim Health (2021), 60(1) : 58-65

DOI:10.36062/ijah.60.1.2021.58-65

\title{
Sex-specific reference intervals for Wistar albino rats: hematology and clinical biochemistry
}

\author{
R. Vigneshwar ${ }^{1 *}$, A. Arivuchelvan ${ }^{1}$, P. Mekala ${ }^{1}$ and K. Imayarasi ${ }^{2}$ \\ ${ }^{1}$ Department of Veterinary Pharmacology and Toxicology, Veterinary College and Research Institute, \\ Tamilnadu Veterinary and Animal Sciences University, Namakkal- 637 002, Tamilnadu, India; \\ ${ }^{2}$ Central Animal Facility, Christian Medical College, Vellore- 632 004, Tamilnadu, India
}

\begin{abstract}
Wistar albino rats are the most commonly used laboratory rats for pharmacological, toxicological and physiological experimental studies. Standardization of reference intervals for clinically important hematological and biochemical parameters would help the researchers to compare their study results using Wistar rats as an experimental animal for evaluating health status and organ functions. In this study, we investigated hematology and common clinical biochemistry values in adult male and female Wistar rats $(n=36)$. Blood samples were collected and analyzed for a complete blood count. Serum samples were analyzed for glucose, total protein, lipid profile, electrolytes, liver function tests, and kidney function tests. Mean values of different parameters among males and females were compared by paired t-test for statistical significance. This study demonstrates significant differences between genders for many parameters such as mean corpuscular hemoglobin concentration (MCHC; $35.43 \mathrm{~g} / \mathrm{dL}$ in male and $38 \mathrm{~g} / \mathrm{dL}$ in female), percentage of monocytes $(7.63 \%$ in male and $6.54 \%$ in female), lipid profile (high levels of triglycerides, LDL and VLDL cholesterols in male), levels of serum sodium (136.81 meq/L in male and $145.62 \mathrm{meq} / \mathrm{L}$ in female) and liver enzymes. These reference values may help to verify results using Wistar rats as a model.
\end{abstract}

Key words: Electrolytes, Glucose, Lipid profile, Liver function, Monocytes

\section{INTRODUCTION}

Laboratory animals have always been used as important investigation tools in biomedical research and pre-clinical toxicity studies. Experiments on the development of new drugs, vaccines, surgical materials, diagnostic kits, safety and toxicity testing of different substances, etc. are possible only with animal models. Despite certain differences between humans and rodents, the similarities are strong enough to give researchers an enormously strong mammalian system to investigate human diseases (Car et al., 2006). Mice, rats, rabbits, and guinea pigs are the commonly used laboratory animals in biomedical research, mice and rats make up approximately $95 \%$ of all laboratory animals. Among rats, Wistar rats are the most commonly used animal in pre-clinical toxicology research. Wistar rats are the commonly selected animal model for a variety of reasons, including short reproductive cycle and lifespan; availability of information regarding their anatomy, biology and physiology; they are mild-tempered, docile, and small in size which facilitate housing and maintenance than Sprague Dawley (SD) rats. They are mammals and their organ systems are very similar to humans in terms of shape, structure and physiology (Hickman et al., 2017; Vigneshwar et al., 2021).

Reference intervals are very important laboratory tools used to make a medical diagnosis, therapeutic management decision, or other physiological assessment in the clinical laboratory (Walter, 1997). The establishment of reference values is necessary for the clinical interpretation of laboratory data. Hematologic and biochemical reference values are critical for assessing the health and disease states related to the diagnosis of blood disorders, infectious diseases, disorders of immune system and metabolism, hormone regulation, and organ

\footnotetext{
*Corresponding Author
} 
function. When these parameters have deviated from their normal homeostatic level, the increase or decrease of their levels or activities could result in diseases (Delwatta et al., 2018). Lack of a normal range of reference values during a research setting under local conditions is often a drawback for beginners; because the accuracy of data from control samples can not be verified at the beginning of a research project, this affects comparisons with data from test samples (Clemo, 1997).

In this context, the hematological and biochemical parameters of Wistar rats are of significance to researchers as they are used to evaluating vital information about the response of the body to different diseases and treatments. These parameters are influenced by a wide range of environmental and physiological factors including diet, age, sex, housing, micro, and macro environment, and management factors, and changes in any of these conditions would affect the reference values of the hematological and biochemical parameters.

Despite different reference values for Wistar rats have been published by researchers from different countries (Boehm et al., 2007; Giknis and Charles, 2008; Kampfmann et al., 2012), those reports are decade old and can be affected by many factors, such as nutrition, animal housing, or management of local conditions. Kampfmann et al. (2012) from Germany, reported that male Wistar rats had significantly higher levels of WBC, RBC, neutrophils, lymphocytes and monocytes compared to female rats and the remaining parameters were statistically non-significant ( $n=68$ for each gender, with a single blood sample per animal drawn from a sublingual vein), whereas Jacob et al. (2017) from Brazil, reported that male Wistar rats had significantly higher levels of WBC, RBC, HCT, neutrophils and lower levels of $\mathrm{MCV}, \mathrm{MCH}, \mathrm{MCHC}$, lymphocytes, and eosinophils than female Wistar rats $(n=70$ for each gender, with seven blood samples per animal on different time points drawn from a tail vein). This could be an epitome of how using the same species, researchers from different countries can detect dissimilarities in the measured parameters based on the age of the animal, site, and frequency of blood collection, analytical procedures, and the number of samples analyzed. Hence, establishing a reference database for clinically important hematological and biochemical parameters of the Wistar rats housed in a conventional animal facility in India will be of great importance for future Indian researchers.

Taking the above points into consideration, this study has been carried out with the following objectives:

1. To estimate the hematological and clinically important biochemical analytes of Wistar rats.

2. To optimize the sex-specific reference intervals for the selected parameters in male and female Wistar rats.

3. To compare the measured hematological and biochemical parameters of Wistar rats with SpragueDawley rats and humans.

\section{MATERIALS AND METHODS Animals}

Apparently, healthy four months old Wistar albino rats of both sex (18 male and 18 female) bred in the animal facility of Veterinary College and Research Institute, Namakkal, Tamilnadu were used for this study. Rats were reared in polypropylene cages under standard management conditions as per the CPCSEA guidelines. They were fed with standard rat chow and purified water ad libitum. The rats were handled humanely throughout the experiment. This experiment was approved by the Institutional Animal Ethics Committee of Veterinary College and Research Institute, Namakkal (IAEC 09/2018).

\section{Sample collection}

All the animals fasted overnight before the collection of blood samples. Animals were anaesthetized with intraperitoneal injection of xylazine $(10 \mathrm{mg} / \mathrm{kg}$ b.wt.) and ketamine (100 $\mathrm{mg} / \mathrm{kg}$ b.wt.) combination. Blood sample of 
$1.2 \mathrm{~mL}$ was collected from each rat by retroorbital bleeding and dispensed as follows: 0.2 $\mathrm{mL}$ of blood in EDTA coated vials for hematological investigations and $1.0 \mathrm{~mL}$ of blood was dispensed in a plain tube, then allowed to stand for 30 minutes at room temperature to clot and centrifuged to harvest serum. The sera were aliquoted and stored at $20^{\circ} \mathrm{C}$ till further analysis. This was repeated for four consecutive weeks, in order to standardize the measurements of selected hematological and biochemical parameters. A total of 72 samples were analyzed for each hematological and biochemical parameter for each gender using 18 animals over the four weeks.

\section{Hematology analysis}

Hematological parameters were analyzed by an automated animal blood cell counter (Horiba, ABX Micros ESV60). The 13 hematology parameters included hemoglobin $(\mathrm{Hb})$, hematocrit (HCT), mean corpuscular hemoglobin $(\mathrm{MCH})$, mean corpuscular hemoglobin concentration (MCHC), mean corpuscular volume (MCV), red blood cell (RBC) count, platelet (PLT) count, white blood cell (WBC) count, and percentages of differential leukocyte count (neutrophils, lymphocytes, monocytes, eosinophils and basophils).

\section{Serum biochemistry analysis}

Fifteen serum biochemical parameters included glucose, lipid profile such as total cholesterol, triglycerides (TG), high-density lipoprotein (HDL-C), low-density lipoprotein (LDL-C), very low-density lipoprotein (VLDL-C), total protein, alanine aminotransferase (ALT), and aspartate aminotransferase (AST) to determine liver function, creatinine, and blood urea nitrogen (BUN) to determine renal function, and serum electrolytes such as calcium, phosphorus, sodium and potassium. Serum biochemical investigations were performed using Span and Tulip diagnostics kits as per the manufacturer's guidelines using a UV double beam spectrophotometer (Systronics 2201). LDL and
VLDL cholesterols were calculated mathematically according to Friedewald's equation (Friedewald et al., 1972). Sodium and potassium levels were estimated by using a flame photometer (Systronics 129) with reference to standard procedures (Pennacchia et al., 1971).

\section{Statistical presentation of data}

The means \pm SEM (standard error of the mean), of the measured parameters, were calculated separately for male and female rats. Mean values of the hematological and biochemical parameters among males and females were compared separately by one-way ANOVA for statistical significance using the SPSS software package. $\mathrm{P}<0.05$ was considered statistically significant. Minimum and maximum values for each parameter were also presented as a range.

\section{RESULTS \\ Reference intervals of hematologic and biochemical analytes}

Measured hematological and biochemical parameters of Wistar rats are given in Tables 1 and 2. Although mean values of hematological parameters of male and female rats were closely similar to each other, based on the statistical comparisons, differences in mean values of MCHC and percentage of monocytes between male and female rats were statistically significant $(\mathrm{P}<0.05)$. Male rats had a significantly higher percentage of monocytes and lower levels of MCHC compared to female rats. Serum glucose, total protein, creatinine and BUN did not show statistically significant differences. In lipid profile, the mean serum total cholesterol and HDL cholesterol were statistically non-significant, whereas triglycerides, LDL-C and VLDL-C differ significantly (high in male rats) between male and female $(\mathrm{P}<0.05)$. The liver enzymes, AST and ALT showed a gender difference (high in female rats) that was statistically significant. Among, the serum electrolytes measured, the difference in sodium level was statistically 
Table 1. Mean and range of measured hematological analytes of Wistar albino rats $(n=36)$

\begin{tabular}{|c|c|c|c|c|}
\hline \multirow[b]{2}{*}{ Analyte } & \multicolumn{2}{|c|}{ Mean \pm S.E.M } & \multicolumn{2}{|c|}{ Range } \\
\hline & Male & Female & Male & Female \\
\hline $\mathrm{WBC}\left(\times 10^{3} / \mathrm{mm}^{3}\right)$ & $4.43 \pm 0.74$ & $3.10 \pm 0.16$ & $3.70-5.80$ & $2.50-3.60$ \\
\hline $\mathrm{RBC}\left(\times 10^{6} / \mathrm{mm}^{3}\right)$ & $7.29 \pm 0.31$ & $6.95 \pm 0.79$ & $6.10-8.50$ & $5.10-8.10$ \\
\hline $\mathrm{Hb}(\mathrm{g} / \mathrm{dL})$ & $13.68 \pm 0.58$ & $15.10 \pm 0.98$ & $11.80-16.20$ & $10.70-17.70$ \\
\hline $\operatorname{HCT}(\%)$ & $38.65 \pm 1.78$ & $37.11 \pm 2.14$ & $32.60-46.20$ & $27.30-48.40$ \\
\hline PLT $\left(\times 10^{3} / \mathrm{mm}^{3}\right)$ & $405.84 \pm 30.97$ & $415.50 \pm 28.19$ & $315-512$ & $330-540$ \\
\hline Lymphocytes \% & $62.26 \pm 1.53$ & $65.88 \pm 2.00$ & $54.90-65.30$ & $59.80-73.90$ \\
\hline Monocytes \%* & $7.67 \pm 0.12$ & $6.67 \pm 0.14$ & $7.00-8.30$ & $5.60-7.30$ \\
\hline Neutrophils \% & $28.51 \pm 1.21$ & $25.46 \pm 1.70$ & $23.40-40.50$ & $20.50-37.50$ \\
\hline Eosinophils \% & $1.30 \pm 0.70$ & $1.82 \pm 1.00$ & $0.30-3.40$ & $0.50-4.50$ \\
\hline Basophils \% & $0.30 \pm 0.20$ & $0.30 \pm 0.30$ & $0-0.80$ & $0-0.80$ \\
\hline MCV (fL) & $54.00 \pm 1.81$ & $53.50 \pm 1.72$ & $51-62$ & $50-60$ \\
\hline $\mathrm{MCH}(\mathrm{pg})$ & $19.11 \pm 0.30$ & $20.34 \pm 0.42$ & $17.70-20$ & $18.70-22$ \\
\hline $\operatorname{MCHC}(\mathrm{g} / \mathrm{dL}) *$ & $35.43 \pm 0.10$ & $38.00 \pm 0.37$ & $32.70-36.20$ & $36.80-40.80$ \\
\hline
\end{tabular}

*Statistically significant parameters $(\mathrm{P}<0.05)$

Table 2. Mean and range of measured biochemical analytes of Wistar albino rats $(n=36)$

\begin{tabular}{lllll}
\hline & \multicolumn{2}{c}{ Mean \pm S.E.M } & \multicolumn{2}{c}{ Range } \\
\cline { 2 - 5 } \multicolumn{1}{c}{ Analyte } & \multicolumn{1}{c}{ Male } & \multicolumn{1}{c}{ Female } & \multicolumn{1}{c}{ Male } & \multicolumn{1}{c}{ Female } \\
\hline Glucose $(\mathrm{mg} / \mathrm{dL})$ & $125.54 \pm 10.32$ & $120.15 \pm 10.92$ & $90.75-180.25$ & $82.68-170.36$ \\
Total protein (g/dL) & $5.82 \pm 0.56$ & $6.18 \pm 0.45$ & $5.10-7.60$ & $5.20-8.20$ \\
Total cholesterol (mg/dL) & $80.12 \pm 3.56$ & $76.83 \pm 5.30$ & $60.52-100.20$ & $62.47-104.13$ \\
TG (mg/dL)* & $64.97 \pm 5.87$ & $41.00 \pm 4.82$ & $32.06-78.24$ & $26.78-65.88$ \\
HDL-C (mg/dL) & $46.16 \pm 2.04$ & $49.89 \pm 4.04$ & $36.78-54.65$ & $37.00-68.73$ \\
LDL-C (mg/dL)* & $24.16 \pm 3.70$ & $19.36 \pm 2.10$ & $15.58-35.09$ & $12.21-27.36$ \\
VLDL-C (mg/dL)* & $12.63 \pm 1.15$ & $8.60 \pm 0.82$ & $6.40-16.50$ & $5.05-13.16$ \\
BUN (mg/dL) & $17.19 \pm 2.70$ & $18.32 \pm 1.60$ & $12.30-20.10$ & $13.5-24.5$ \\
Creatinine (mg/dL) & $0.49 \pm 0.10$ & $0.42 \pm 0.10$ & $0.30-0.60$ & $0.30-0.60$ \\
ALT (IU/L)* & $34.33 \pm 9.34$ & $46.75 \pm 7.27$ & $24.13-67.45$ & $29.34-72.16$ \\
AST (IU/L)* & $64.78 \pm 12.66$ & $76.12 \pm 13.53$ & $55.45-98.10$ & $62.75-126.65$ \\
Calcium (mg/dL) & $8.47 \pm 0.34$ & $8.13 \pm 0.24$ & $7.50-10.50$ & $7.40-9.00$ \\
Phosphorus (mg/dL) & $4.34 \pm 0.23$ & $4.78 \pm 0.58$ & $3.50-7.50$ & $3.80-8.00$ \\
Sodium (meq/L)* & $136.81 \pm 2.20$ & $145.62 \pm 5.38$ & $130.92-148.76$ & $140.34-155.82$ \\
Potassium (meq/L) & $6.36 \pm 0.34$ & $5.87 \pm 0.19$ & $4.20-7.80$ & $4.00-7.50$ \\
\hline
\end{tabular}

*Statistically significant parameters $(\mathrm{P}<0.05)$

significant (high in female rats), and the levels of other electrolytes calcium, phosphorus and potassium were statistically non-significant.

\section{Comparison of reference intervals}

The recorded reference intervals were compared with results from Sprague-Dawley (SD) 
rats and humans (Indian population) from literature and presented in Table 3, detailed sexspecific reference intervals were given for all analytes. SD rats showed higher reference intervals for WBC, RBC, HCT, platelets and lymphocytes than in Wistar rats and humans. Many analytes were similar between Wistar rats and humans, but rodents (both SD rats and
Wistar rats) had higher lymphocyte reference intervals and lower neutrophils reference intervals compared to humans. Rodents also showed higher platelet, AST and glucose reference intervals, and lowest intervals for $\mathrm{MCV}$ and $\mathrm{MCH}$ compared to humans. In contrast to rodents, humans had higher reference intervals for total cholesterol,

Table 3. Mean reference values in male and female Wistar rats from the present study in comparison with SD rats and humans (Indian population) from literature

\begin{tabular}{lllllll}
\hline & \multicolumn{2}{c}{ Wistar rats } & \multicolumn{2}{c}{ S.D rats $^{\#}$} & \multicolumn{2}{c}{ Humans $^{\# \#}$} \\
\cline { 2 - 7 } \multicolumn{1}{c}{ Analyte } & \multicolumn{1}{c}{ Male } & Female & \multicolumn{1}{c}{ Male } & \multicolumn{1}{c}{ Female } & Male & Female \\
\hline WBC $\left(\times 10^{3} / \mathrm{mm}^{3}\right)$ & $3.7-5.8$ & $2.5-3.6$ & $9.7-12.9$ & $7.0-10.6$ & $4.0-10.4$ & $4.2-9.8$ \\
RBC $\left(\times 10^{6} / \mathrm{mm}^{3}\right)$ & $6.1-8.5$ & $5.1-8.1$ & $7.8-10.2$ & $7.2-8.1$ & $4.5-5.5$ & $3.5-5.2$ \\
Hb $(\mathrm{g} / \mathrm{dL})$ & $11.8-16.2$ & $10.7-17.7$ & $11.5-18.4$ & $11.7-17.3$ & $12.3-17$ & $9.9-14.3$ \\
HCT $(\%)$ & $32.6-46.2$ & $27.3-48.4$ & $38.9-54.9$ & $35.2-49.8$ & $37-51$ & $30-43$ \\
PLT $\left(\times 10^{3} / \mathrm{mm}^{3}\right)$ & $315-512$ & $330-540$ & $765-1029$ & $787-1021$ & $130-380$ & $130-420$ \\
Lymphocytes \% & $54.9-65.3$ & $59.8-73.9$ & $61-86$ & $55-86$ & $18-45$ & $18-45$ \\
Monocytes \% & $7.0-8.3$ & $5.6-7.3$ & $1-4$ & $1-4$ & $2-10$ & $2-9$ \\
Neutrophils \% & $23.4-40.5$ & $20.5-37.5$ & $13-36$ & $13-36$ & $42-74$ & $44-75$ \\
Eosinophils \% & $0.3-3.4$ & $0.5-4.5$ & $0-2$ & $0-2$ & $1-8$ & $1-8$ \\
Basophils \% & $0-0.8$ & $0-0.8$ & $0.3-0.5$ & $0.2-0.5$ & $0-0$ & $0-0$ \\
MCV (fL) & $51-62$ & $50-60$ & $45-60$ & $48-59$ & $78-97$ & $72-96$ \\
MCH (pg) & $17.7-20$ & $18.7-22$ & $18-21$ & $17-20$ & $26-33$ & $23-32$ \\
MCHC (g/dL) & $32.7-36.2$ & $36.8-40.8$ & $31-35$ & $32-36$ & $31-36$ & $30-35$ \\
Glucose (mg/dL) & $90-180$ & $82-170$ & $121-197$ & $120-186$ & $78-110$ & $76-108$ \\
Total protein (g/dL) & $5.1-7.6$ & $5.2-8.2$ & $6.0-7.1$ & $6.4-7.5$ & $6.8-8.5$ & $6.7-8.5$ \\
Total cholesterol (mg/dL) & $60-100$ & $62-104$ & $55-89$ & $66-97$ & $115-254$ & $117-252$ \\
TG (mg/dL) & $32-78$ & $26-65$ & $62-92$ & $51-75$ & $55-267$ & $52-207$ \\
HDL-C (mg/dL) & $36-54$ & $37-68$ & $29-42$ & $32-63$ & $25-61$ & $29-70$ \\
LDL-C (mg/dL) & $15-35$ & $12-27$ & $18-42$ & $14-38$ & $60-176$ & $61-170$ \\
BUN (mg/dL) & $12-20$ & $13-24$ & $10-16$ & $11-17$ & $13-35$ & $11-31$ \\
Creatinine (mg/dL) & $0.3-0.6$ & $0.3-0.6$ & $0.2-1.2$ & $0.2-1.2$ & $0.7-1.2$ & $0.6-0.9$ \\
ALT (IU/L) & $24-67$ & $29-72$ & $27-46$ & $25-45$ & $15-74$ & $10-37$ \\
AST (IU/L) & $55-98$ & $62-126$ & $77-110$ & $72-116$ & $14-42$ & $12-37$ \\
Calcium (mg/dL) & $7.5-10.5$ & $7.4-9.0$ & $9.6-10.9$ & $9.6-11.2$ & $8.5-10.5$ & $($ combined) \\
Phosphorus (mg/dL) & $3.5-7.5$ & $3.8-8.0$ & $7.0-9.5$ & $5.6-8.6$ & $2.8-4.7$ & $($ combined) \\
Sodium (meq/L)* & $130-148$ & $140-155$ & $141-149$ & $141-148$ & $135-146$ & $($ combined) \\
Potassium (meq/L) & $4.2-7.8$ & $4.0-7.5$ & $4.6-6.1$ & $4.3-5.9$ & $3.8-5.0$ & $($ combined) \\
\hline
\end{tabular}

\#Reference data for SD rats (Giknis and Charles, 2006; He et al., 2017)

\# Reference data for humans (Sairam et al., 2014; Shah et al., 2018) 
triglyceride, LDL cholesterol, and total protein. In particular, the range for AST and ALT in human males was significantly higher than that in females, but the opposite occurred in Wistar rats. Reference intervals of serum electrolytes were similar between rodents and humans.

\section{DISCUSSION}

The current study was conducted to optimize the sex-specific reference intervals for the selected hematological and biochemical analytes of the adult Wistar albino rats which are commonly used in pre-clinical toxicity studies. In hematological parameters, sex differences were observed in one of the RBC indices, $\mathrm{MCHC}$, and also in the percentage of monocytes. The findings were in agreement with the results of Kampfmann et al. (2012). Male usually have a slightly higher count of monocytes than females, this variation might be related to the sex differences in the development of immunocompetence and immune surveillance system (Wolford et al., 1987; Katoh et al., 1992). The other hematological parameters were statistically nonsignificant between male and female rats. We observed similarities and differences among our estimated values compared with those of international researchers (Giknis and Charles, 2008; Kampfmann et al., 2012; Jacob et al., 2017). While mean HCT, platelets, and percentage of lymphocytes values were lower in the current study than those of Giknis and Charles (2008), mean MCV, MCH, MCHC, and percentage of monocytes were high compared to the values for male and female Wistar rats determined by Jacob et al. (2017). This can be attributed to differences in age, micro and macro living environments of the animals, site, and frequency of blood collection and analytical procedures. The mean $\mathrm{WBC}, \mathrm{RBC}, \mathrm{Hb}, \mathrm{MCV}$, $\mathrm{MCH}$ and $\mathrm{MCHC}$ values were opined with the findings of Giknis and Charles (2008), and the mean WBC, RBC, and differential leukocyte count values were opined with the findings of Kampfmann et al. (2012).

Concerning biochemical parameters, females generally showed higher values than males, in agreement with previously reported studies for AST, ALT, BUN, HDL and sodium, whereas male rats had a significantly higher level of triglycerides, LDL-C, and VLDL-C (Teixeira et al., 2000; Boehm et al., 2007; Giknis and Charles, 2008), the remaining parameters were statistically non-significant. The gender differences for several biochemical analytes might be individual differences, which may be related to genetics, sex hormones and enzyme activities but they could not be related to the micro and macro living environments of the animals, the source of food and water supply, and type of bedding material, as all these were common to all the animals used for this study.

Our lipid profile values are opined with the results of Ihedioha et al. (2013) who optimized the reference values for the serum lipid profile of Wistar albino rats of varied ages and sexes. Hydroxymethylglutaryl-CoA reductase (HMGCoA reductase) is the principal rate-limiting enzyme of cholesterol biosynthesis. The expression level of this enzyme is controlled by many factors that in turn regulate cholesterogenesis and cellular cholesterol homeostasis. Low levels of HMGCoA reductase expression in females indicated that cholesterol synthesis was physiologically lower in females than in males (Friesen and Rodwell, 2004; De Marinis et al., 2008).

Female rats showed distinctly higher AST and ALT activity, which are commonly used liver injury biomarkers, but not only specific to the liver. ALT is also considered as a biomarker to rule out non-alcoholic fatty acid liver disease and is also associated with insulin resistance, metabolic syndrome, and type 2 diabetes. The differences in transaminase levels between rodents and humans might be explained by the differences in anatomy such as lack of gallbladder in rats, diverse protein synthesis, and metabolic liver function (Boehm et al., 2007; He et al., 2017). Since these markers are not specific to the liver, it is hard to explain the reason for these higher values in females. 
In the current report, male and female rats did not show significant differences for the values of serum electrolytes such as calcium, phosphorus and potassium, but the level of sodium differs significantly. Boehm et al. (2007) found gender differences for all the electrolytes; phosphorus estimation was not carried out in their study. This can be attributed to differences in the sample size, analytical procedures, instruments and reagents, and also the method of statistical analysis, which may affect the accuracy of the test results.

To date, published reference intervals for hematological and clinical biochemistry data in Wistar rats are very few. The current study was conducted with the objective of establishing a reference database for hematological and selected biochemical parameters of the Wistar rats reared in the conventional animal facility in

\section{REFERENCES}

Boehm O, Zur B, Koch A, Tran N, Freyenhagen R et al., 2007. Clinical chemistry reference database for Wistar rats and C57/BL6 mice. Biol Chem, 388(5): 547-554, doi: 10.1515/BC.2007.061

Car BD, Eng VM, Everds NE and Bounous DI, 2006. Clinical pathology of the rat. In: The Laboratory Rat, $2^{\text {nd }}$ edn. (Suckow MA, Weisbroth SH and Franklin CL, edn.). Elsevier, London (UK), pp 127-145

Clemo FAS, 1997. Response to utility of clinical pathology reference ranges in pre clinical safety studies. Toxicol Pathol, 25 (6): 650

De Marinis E, Martini C, Trentalance A and Pallottini $\mathrm{V}, 2008$. Sex differences in hepatic regulation of cholesterol homeostasis. J Endocrinol, 198(3): 635-643, doi: 10.1677/JOE-08-0242

Delwatta SL, Gunatilake M, Baumans V, Seneviratne MD, Dissanyaka MLB et al., 2018. Reference values for selected hematological, biochemical and physiological parameters of Sprague Dawley rats at the Animal House, Faculty of Medicine, University of Colombo, Sri Lanka. Animal Model Exp Med, 1(4): 250-254, doi: 10.1002/ ame2.12041

Friedewald WT, Levy RI and Fredrickson DS, 1972. Estimation of concentration of low density lipoprotein cholesterol in plasma without use
India. In this study, we clearly demonstrate that hematological parameters of male and female Wistar rats are almost similar except few parameters whereas many biochemical parameters showed gender differences. Therefore, it is necessary to partition the reference intervals based on sex to more precisely evaluate the animal health and also to compare the results of the experimental group with the control group in pre-clinical toxicology studies using Wistar rats as an animal model.

Conflict of interest: Authors have no conflict of interest in this study.

\section{ACKNOWLEDGEMENTS}

We thank Dr. Murugesan, The Head, Department of Veterinary Pharmacology and Toxicology, for his valuable guidance throughout the study.

of preparative ultracentrifuge. Clin Chem, 18(6): 499-502

Friesen JA and Rodwell VW, 2004. The 3-hydroxy-3methylglutaryl coenzyme-A (HMG-CoA) reductases. Genome Biol, 5(11): 248, doi: $10.1186 / g b-2004-5-11-248$

Giknis MLA and Charles BC, 2006. Clinical laboratory parameters for $\mathrm{Crl}$ : CD (SD). Charles River Laboaratories, UK, pp 1-14

Giknis MLA and Charles BC, 2008. Clinical laboratory parameters for $\mathrm{Crl}$ : WI (Han). Charles River Laboaratories, UK, pp 1-14

He Q, Su G, Liu K, Zhang F, Jiang Y et al., 2017. Sexspecific reference intervals of hematologic and biochemical analytes in Sprague-Dawley rats using the nonparametric rank percentile method. PLoS One, 12(12): e0189837, doi: 10.1371/ journal.pone.0189837

Hickman DL, Johnson J, Vemulapalli TH, Crisler JR and Shepherd R, 2017. Commonly Used Animal Models. In: Principles of Animal Research for Graduate and Undergraduate Students. $1^{\text {st }}$ edn. (Suckow M and Stewart K, eds.) Elsevier Academic Press, USA, pp 117-175, doi: 10.1016/B978-0-12-802151-4.00007-4

Ihedioha JI, Uneke OA and Ihedioha TE, 2013. Reference values for the serum lipid profile of 
albino rats (Rattus norvegicus) of varied ages and sexes. Comp Clin Path, 22 (1): 93-99, doi: 10.1007/s00580-011-1372-7

Jacob FW, Lima CC, Paunksnis MRR, Silva AA, Perilhao MS et al., 2017. Reference database of hematological parameters of growing and aging rats. Aging Male, 21(2): 145-148, doi: 10.1080/ 13685538.2017.1350156

Kampfmann I, Bauer N, Johannes S and Moritz A, 2012. Differences in hematologic variables in rats of the same strain but different origin. Vet Clin Pathol, 41(2): 228-234, doi: 10.1111/j.1939165X.2012.00427.x

Katoh M, Ozawa M, Akima T, Ikeda H, Fujii T et al., 1992. Hematological and serum biochemical changes with age in term fetuses, offspring and dams in normal Sprague-Dawley rats. Jikken Dobutsu, 41(3): 329-337, doi: 10.1538/ expanim1978.41.3_329

Pennacchia G, Bethune VG, Fleisher M and Schwartz MK, 1971. Automated flame photometry of serum sodium and potassium. Clin Chem, 17(4): 339-340

Sairam S, Domalapalli S, Muthu S, Swaminathan J, Ramesh VA et al., 2014. Hematological and biochemical parameters in apparently healthy Indian population: defining reference intervals.
Indian J Clin Biochem, 29(3): 290-297, doi: 10.1007/s12291-013-0365-5

Shah SAV, Ichihara K, Dherai AJ and Ashavaid TF, 2018. Reference intervals for 33 biochemical analytes in healthy Indian population: C-RIDL IFCC initiative. Clin Chem Lab Med, 56(12): 2093-2103, doi: 10.1515/cclm-2018-0152

Teixeira MA, Chaguri LG, Carissmi AS, de Souza NL, Mori CMC et al., 2000. Hematological and biochemical profile of rats (Rattus norvegicus) kept under micro environmental ventilation system. Braz J Vet Res Anim Sci, 37(5): 341347, doi: 10.1590/S1413-95962000000500001

Vigneshwar R, Arivuchelvan A and Mekala P, 2021. Thyrogenic, hypolipidemic and antioxidant effects of Bacopa monnieri (Brahmi) on experimental hypothyroidism in rats. $\mathrm{J}$ Pharmacogn Phytochem, 10(1): 454-458

Walter GL, 1997. More perspectives on hysterical controls for clinical pathology. Toxicol Pathol, 25(6): 651, doi: 10.1177/019262339702500620

Wolford ST, Schroer RA, Gallo PP, Gohs FX, Brodeck $\mathrm{M}$ et al., 1987. Age-related changes in serum chemistry and hematology values in normal Sprague-Dawley rats. Fundam Appl Toxicol, 8(1): 80-88, doi: 10.1016/0272-0590(87) 90102-3

Received - 01.05.2021, Accepted - 26.05.2021, Published - 01.06.2021

Section Editor: Dr. A. K. Patra, Associate Editor 